\title{
Growth Rules: Quasi-Historical Development of Urban Districts
}

\author{
PETER LYNCH \\ City College of CUNY \\ USA
}

\section{INTRODUCTION}

Our research asks how laws could guide the development of an urban district, without reference to a pre-established street plan, zoning plan, or property subdivision. Could urban development be regulated as a self-organizing system, where a succession of local events, constrained by simple rules, resolves the large-scale structure? We believe that an incremental planning process could help give new districts a sense of particularity, space and order- a sense of place sui generis, which seems missing from so much postwar urban development.

We could take a medieval city like Siena as an example of urban self-organization, if we could agree that 1) it has a particular spatial order, a recognizable but unforseeable logic in the disposition of places and routes; 2) this order was arrived at without a plan conception, through the accumulation of local changes, held together by formal and informal regulation; 3) the original settlement pattern played an instrumental, but not a definitive, role in the medieval layout (Benevolo 258). Of course, to concede to all this is to concede the argument. Camillo Sitte, for one, would not agree- despite his sympathetic attitude towards unplanned urban growth. He attributed the beauty of the medieval city to a lost artistic tradition-a tragic diagnosis, in our opinion, since it implies that an improvement in contemporary urban planning must await a collective awakening of artistic consciousness (Collins Chapter XI and Appendix II). But the strange familiarity and appropriateness of medieval streets could be an artifact, without being consciously produced. The track of a pathway through a derelict quarter, setting the bed of a new road... the detour around an obstacle then made permanent... the widening of a thoroughfare, constricted by objects now gone... Even with the overturning of circumstance, the paths retain the character of evidence; perhaps because we recognize the signature of an intention in the curve itself. Patterns of activity become conduits of activity, like the self-organizing process of the brain network, in which pathways are etched by the initial stimulation of a uniformly interconnected field.

Rather than asking about the possibility of a self-orga- nized city plan, one might more reasonably wonder if there is any circumstance other than extemporized change and adjustment, historically accumulated or internally coordinated, which could arrive at urban character and sense of place. In our research we are asking if evolution can be structured, in an objective, cumulative, non-trial-and-error way, according to a set of rules, which could arrive at an urban configuration in decades or years rather than centuries.

This work was initially directed towards a proposal for Ørestad, a new district to be built just south of historic Copenhagen. In 1994 an international competition requested proposals and strategies for implementation, presuming private development over a thirty-year period.'

\section{SELF-ORGANIZING SYSTEMS}

The neural network is not a perfect analogy for our problem, since its initial state is generic, and describes the state of all possible outcomes. At the site for a city the particular terrain, rudimentary and adjacent patterns of settlement, infrastructural demands, etc. describe initial conditions for growth, without anticipating a set of possible configurations. A well chosen example for our purpose would need to show small-scale elements interacting to form a large-scale order. We are interested in cell mitosis, for example; in which microtubules, spontaneously assembled and extended, sort and separate the chromosomes (Glover). Or again, we are struck by the behavior of some mathematical systems such as cellular automata, which settle unpredictably upon monotonic, patterned, or chaotic states (Dewdney). In both of these examples the initial conditions are simple; they are instruction-sets, not descriptions of large-scale structures. In the first example, the microtubule-assembly has only one structural outcome. In the second example, outcomes vary radically. These two systems suggest limits for a set of urban growth rules; for the design of a city is neither a technical, nor a formal, problem. Our goal is not to determine the set of laws which would direct random behavior towards a particular structure, nor to determine a system which might settle insignificantly, or disastrously, upon one of a number of regimes; but rather to describe laws which allow events to 
construct one of many outcomes, all substantially and meaningfully different, and all fulfilling the functional and practical requirements of a city district.

In an urban-planning approach of this kind, each private building project would incrementally resolve the overall plan. For the result to be more than a patchwork, a disaster, or a fait accompli, circuitously realized, the status quo at any point would have to restrict and project future choices, while holding many alternatives open. The range of possibilities would begin to narrow as more and more positions were taken. Every possible state would need to have at least one resolution. Furthermore, if we accept the privately financed, risk/reward engine of development, then any reform of the planning process would have to preserve the profitability of development. This implies that 1 ) the experiential character of the district should be known beforehand, written into the growth rules themselves; 2 ) it should be possible to guarantee a logical and prudent organization, and to rationalize the pattern of settlement if development of the district is arrested at any stage; 3 ) an accurate means must exist to estimate and regulate the total density of development.

\section{THE RULE SET}

In lieu of a-priori street, lot, or zoning plans, the growth rules would describe frontage, dimension, and adjacency requirements for any buildable site, to be staked out on the ground and checked against a post-facto register of commitments and existing conditions. The lack or undesirability of available sites would compel individuals to project new sites, in accordance with the requirements. Growth rules could establish light and air, open space, setback, density, and curb cut requirements, etc., as per a zoning resolution; but the rules would be formulated in such a way that they would also supply the necessary armature for incremental growth.

In our system, all buildable sites must front on an existing or projected right-of-way. Rights-of-way are divided into two independent networks - vehicular streets and pedestrian/bicycle ways - which have equal status, as far as building orientation is concerned. All sites fronting one network are required to have secondary access to the complementary network. If a site fronts a street, then it must be connected to a bikeway via a common block interior; and vice versa for lots with bikeway frontage. Besides allowing settlements greater freedom vis-à-vis the automobile, the double-network rule also encourages the formation of open blocks, as shown in figure 1. Most importantly, this rule gives each step latitude for invention, resists caprice, and encourages compact configurations. A certain amount of internal resistance is necessary, it seems, to configure a system which resists willfulness on one hand, and determinism on the other.

Dimensional and geometric requirements for rights-ofway are easily codified; the most significant morphologically is the restrictin of cumulative frontage, including gaps,

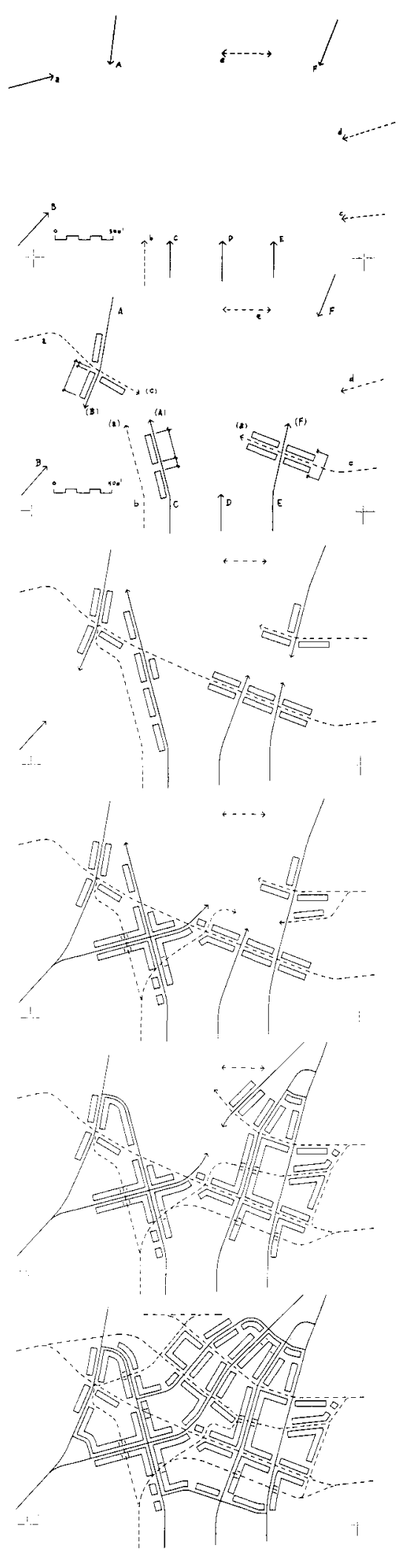

Fig. 1. Hypothetical district; initial conditions and diagrammatic evolution. Solid lines are streets, dashed lines are bikeways. 
to 250 meters or less, measured between existing/projected cross-streets or -ways. In addition, unclaimed "corridors" of land must be spaced $90 \mathrm{~m}$ or less along the block face. These rules discourage strip development, allow for future densification or subdivision, and ensure that the settlement pattern is always block-based.

The requirements for legitimate projections of streets and bikeways are key to the entire growth-rule system. When a developer extends existing rights-of-way, in order to open up a site for development, he/she commits to all (or to a consistently determined part) of the cost entailed by extending the rights-of-way to the furthest end of the purchased site. Construction of roads etc. may not actually proceed in these stages; but the developer must take responsibility for the completion of his/her link, and is "paying by the mile." No new link may be constructed which does not directly connect to an existing right-of-way or to a legitimate destination. In addition, the "free end" of the extension must be projected towards a legitimate destination, via a possible and direct route. ${ }^{2}$ It is important to say that the completion of all projected rights-of-way is a morphological obligation, but one which falls upon future developers; and that each street or bikeway may be projected to any legitimate destination, along any legitimate route, as long as all rules are followed and all obligations to-date are observed. ${ }^{3}$ Projection of possible routes at every step of development insures that at least one resolution of the status quo in fact exists, without tying the future to that scenario. As development of the district reaches its limit, the field of possible routes begins to dwindle, and the final projection becomes de facto the master plan.

Since pedestrian/bicycle travel and automobile travel, ideally, have distinct purposes and scales, their legitimate destinations are defined differently. Bikeways may terminate at local commercial nodes, institutions, etc. within the district; but all primary streets must lead beyond the district. For both systems, a requirement exists that every right-ofway be unique, that is, be the only route which connects two given destinations. At one stroke these rules eliminate the redundant routes of a typical urban grid and the branching routes of a typical suburban subdivision. The resulting network would more closely resemble the pattern of rural high roads, at a smaller scale, and would hopefully maintain their individual character. A meaningful street or way is one which leads somewhere, as Edwin S. Brierley so clearly said in his ACSA Conference paper presentation. After the primary routes are completed end-to-end, they in turn become legitimate destinations for unique secondary roads, and so on.

\section{DENSITY CONTROLS}

An accurate means of estimating and optimizing the density of development, regardless of the evolving configuration, must exist at every stage of development. Without a preestablished total area of developable land, density becomes a difficult matter to control.
Initially, the permitted floor area ratio for every site could be calculated by estimating the maximum portion of the district which could be claimed as a site, given the geometric restrictions of the rule set, and dividing it into the allowable gross floor area for the district as a whole. ${ }^{4}$

As more sites are claimed for development the estimate of buildable area becomes more accurate, and the f.a.r. is increased to compensate for less-than-maximum coverage in already developed areas. ${ }^{5}$ To prevent subsequently developed lots from having a higher density than initially developed lots, which would be a meaningless condition, an increase in the f.a.r. would also apply retroactively to sites previously built upon.

Spatial, architectural and infrastructural imperatives fix an absolute limit to density, and effectively cap the f.a.r. for a given use. To minimize the possibility that an unfolding plan configuration would effectively "lock in" a maximum possible development elow the maximum allowed for the district, the geometry of the rule-set would have to imply minimum, as well as maximum, possible coverage; and this minimum coverage, multiplied by the maximum f.a.r. architecturally acceptable, would have to equal or exceed the gross area of development permitted for the district as a whole.

In addition to its bookkeeping role, the floating f.a.r. also ensures that the density of neighborhoods gradually increases as development accumulates and the capacity of the infrastructure increases. ${ }^{6}$

\section{BUILDING TYPES, URBAN SPACES}

The proposed rules are only of abstract interest, unless they can encourage beautiful, livable patterns of settlement an inhabitation.

As described so far, the rules encourage a purposeful orientation of routes, inflected by changes of circumstance; a free and lively weave of streets and ways; and the shaping of common block interiors. Even the floating f.a.r. can be turned to our spatial benefit. Our rules require front-lot-line development, with no setback, as a prerequisite for supplemental back-lot development, and reduce the relative bulk of rear buildings, both in height and in frontage. This encourages a layered definition of interior block spaces, in contrast with the street walls along block fronts.

Simple rules could permit the extension of existing or proposed boulevards, commercial streets, parkways; could define the zones of commercial ground-floor use, within a specified radius of transport nodes; and could even be written to give one-way streets morphological distinction. But the introduction of different building types, with different height and density requirements, is also essential for a truly urban district; and the regulation of this, given undifferentiated land values, is potentially more complex.

To maintain the possibility and accuracy of density calculations, higher-density development could be considered as an overlay. A fixed percent of the gross area allowed 

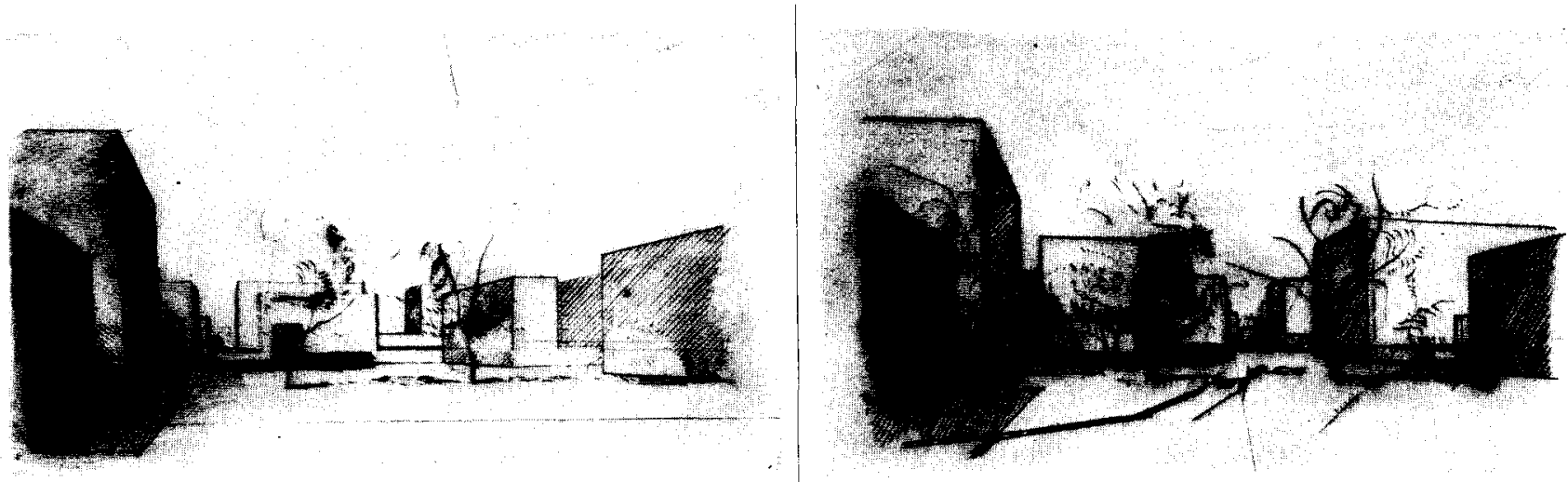

Fig. 2. Hypothetical block interior; early development... ... late development.

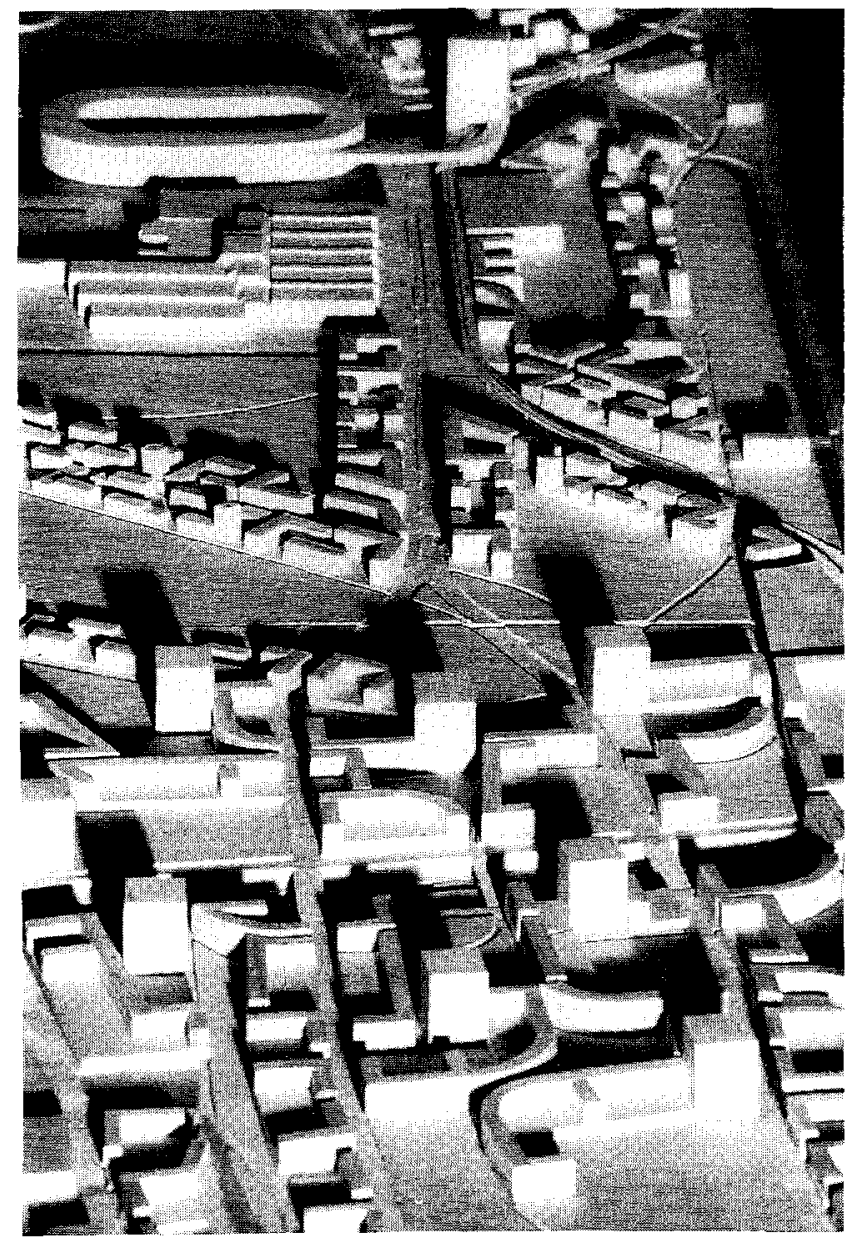

Fig. 3. View of Ørestad proposal; commercial/institutional area in foreground.

for the site would be set aside in the initial rule set. The title to higher-density development would be purchased, and permitted only at sites with certain plan configurations. For example, in our Ørestad proposal all commercial and institutional development is restricted to sites adjoining a common plaza space of certain minimum dimensions; and plazas can only be located at branches in the bicycle/pedestrian network.
A "tower" type, with limited footprint and absolute maximum height, can be regulated spatially in an elegant way. We propose that each tower project an inverted setback cone, which establishes a height ceiling for subsequent tower development in the vicinity (insert Figure 4 in right margin, or at end of text). The cone preserves light and air exposure; directs the assembly of tower clusters in a quasi-compositional way, by defining large spatial volumes; and allows a calculation of maximum volume (and floor area) of tower development for the entire district. Other rules, or a generalization of these, could introduce other building envelopes, and pair permitted building types with other plan configurations.

\section{CONCLUSION}

At least at this schematic level, the formulation of a set of growth rules as a guide to urban development seems promising and possible. Outcomes of test-runs, applied to the Ørestad district, had the inflection and local specificity of historically evolved plans, with a deep integration of green and built, public and private, spaces. The work suggests that this general approach can also be applied to non-urban projects - subdivisions, research centers, office parks. For example, the additive character of the "pastoral" office park lends itself to an incrementally developed circulation system. An initial "constellation" of site-to-site vehicle links, once overloaded, can be hooked up to ramifying one-way express roads - inbound in the morning, outbound in the evening. Traffic in the contrary direction is handled by the original site-to-site network. In a system of this type, peak traffic to and from all destinations must pass only one or two traffic lights; paved area is reduced, for an increased traffic capacity; and expansion of the network is straightforwardly accomplished.

Work along these lines might find exciting sources and inspiration in other fields of research. Biology, geometry of "turtle" and L-systems, computer science, and systems engineering have developed remarkable methods of nonplan-based organization and analysis. For us, it seems no coincidence that the conceptual tools have appeared just when the problem we are facing, the unpremeditated development of order, most seriously calls for a radical solution. 

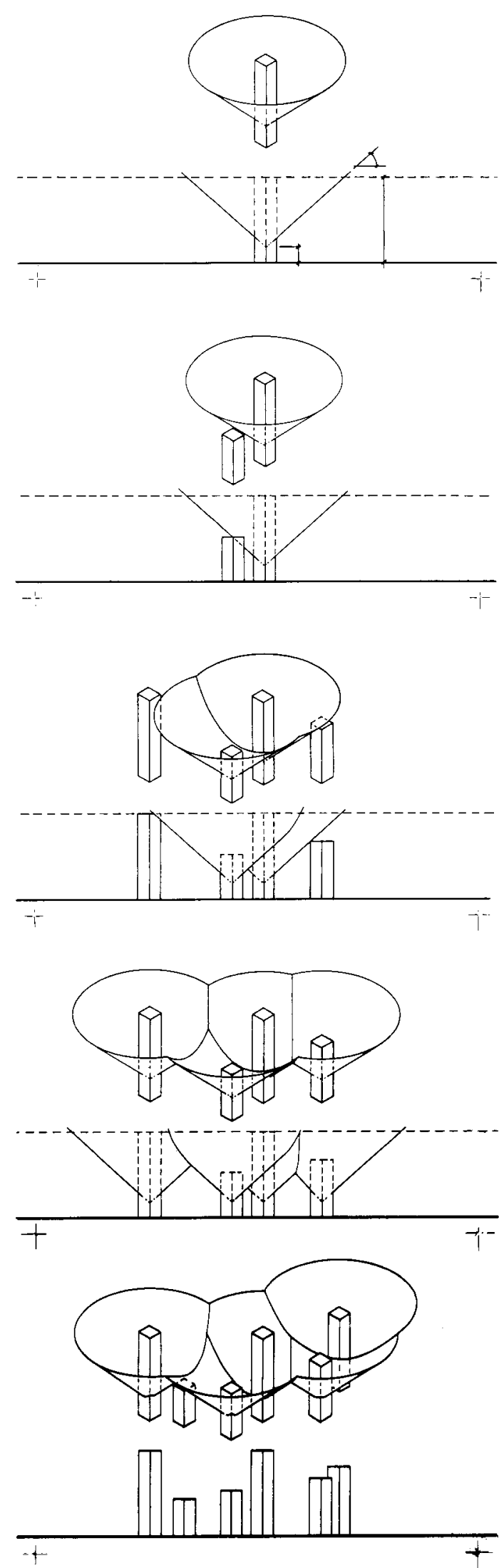

Fig. 4. Setback cone for tower; hypothetical development of tower cluster.

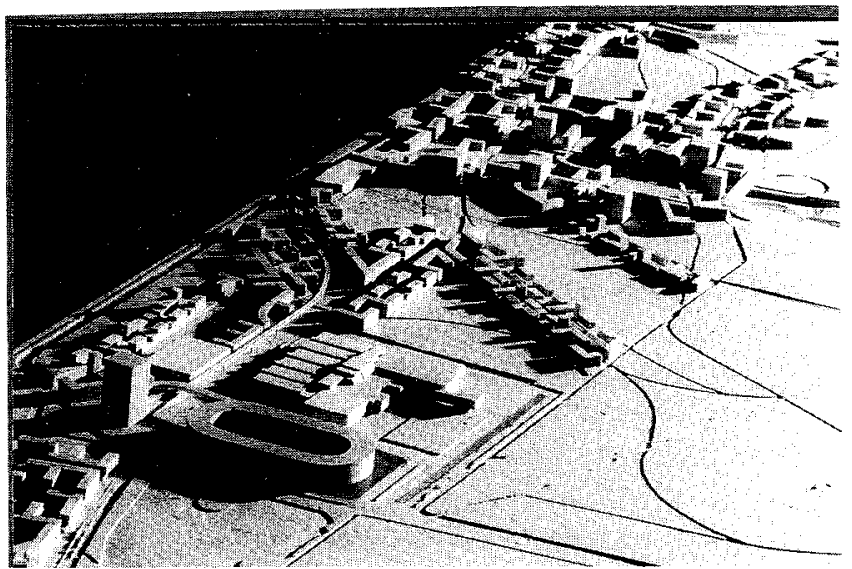

Fig. 5. Partial vew of Ørestad proposal.

\section{NOTES}

${ }^{1}$ Ørestad Competition entry by Peter Lynch with Cory Denningham, Dagmar Frinta, Sabine Fischer, Jan Kinsbergen, Chris and Bill Sharples, Roland Sum, and Lynnette Widder.

${ }^{2}$ For example: a direct route is one for which the maximum difference in angular bearing between any two vectors tangent to the established and projected route is $\pm 100^{\circ}$ for streets, $\pm 140^{\circ}$ for bikeways.

${ }^{3}$ The review of developers proposals, including projections of the street and bikeway networks, is conceived as a public process. A great advantage of the growth-rule approach is its accountability; major infrastructural decisions are open to the review of citizens, including current district occupants. At the same time, the growth rules establish objective criteria for the review/approval/appeal process, which is essential to prevent arbitrariness and xenophobia.

${ }^{4}$ For example: since, according to the rule set, rights-of-way are at least $20 \mathrm{~m}$ wide, lots are at most 45 deep, each lot reserves a part of the common block interior equal to at least one-fourth the lot area, and continuous frontage may not exceed $90 \mathrm{~m}$, the maximum site coverage, expressed as the percent of total land assignable as a lot, would be $(2 \times 90 \times 45) /((2 \times \times 45(1.25)+20)$ $((90+20))=56 \%$. If the gross f.a.r. allowed for the district as a whole is 1.00 , then an individual lot could initially be developed at $1.00 / 0.56$, or 1.80 f.a.r.

${ }^{5}$ For example: if one-quarter of the same district is subsequently developed at a coverage of $30 \%$, then the estimated maximum possible coverage for the district as a whole would become $(0.25(0.30)+(0.75(0.56)=50 \%$, and the f.a.r. would rise to 2.0 .

6 The floating f.a.r. encourages initial development to proceed in stages. Densification could be undertaken after the feasibility of the district is more assured. If the offering price of land is a function of the f.a.r. at the moment, then the land authority is effectively shouldering some of the risk of initial development. This could be considered an advantage or a disadvantage.

\section{REFERENCES}

Benevolo, Leonardo, trans. Geoffrey Culverwell. The History of the City. Cambridge: MIT Press, 1981.

Collins, George R. and Christiane Crasemann Collins. Camillo Sitte: The Birth of Modern City Planning. New York: Rizzoli, 1986.

Glover, David M., et al. "The Centrosome." Scientific American June 1993: p. 62-68.

Dewdney, A. K. "The cellular automata programs that create wireworld, rugworld and other diversions." Scientific American Jan. 1990: p. 146-149. 